\title{
Serum steroid profiling by isotopic dilution-liquid chromatography-mass spectrometry: Comparison with current immunoassays and reference intervals in healthy adults
}

\author{
Flaminia Fanelli ${ }^{a}$, Ilaria Belluomo ${ }^{a}$, Valentina D. Di Lallo ${ }^{a}$, Gaia Cuomo ${ }^{a}$, Rosaria De Iasio ${ }^{b}$, \\ Margherita Baccini ${ }^{\mathrm{a}}$, Elena Casadio ${ }^{\mathrm{a}}$, Bruno Casetta ${ }^{\mathrm{c}}$, Valentina Vicennati ${ }^{\mathrm{a}}$, \\ Alessandra Gambineri ${ }^{\mathrm{a}}$, Gabriele Grossi ${ }^{\mathrm{b}}$, Renato Pasquali ${ }^{\mathrm{a}}$, Uberto Pagotto ${ }^{\mathrm{a}, *}$ \\ a Endocrinology Unit and Centre of Applied Biomedical Research, Department of Clinical Medicine, S.Orsola-Malpighi Hospital, \\ Alma Mater Studiorum, University of Bologna, Italy \\ ${ }^{\mathrm{b}}$ Central Laboratory, S.Orsola-Malpighi Hospital, Bologna, Italy

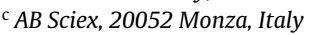

\section{A R T I C L E I N F O}

\section{Article history:}

Received 4 September 2010

Received in revised form

17 November 2010

Accepted 17 November 2010

Available online 26 November 2010

\section{Keywords:}

Tandem mass spectrometry

Immunoassay

Reference interval

\begin{abstract}
A B S T R A C T
Background: The simultaneous, rapid and reliable measurement of a wide steroid panel is a powerful tool to unravel physiological and pathological hormone status. Clinical laboratories are currently dominated by high-throughput immunoassays, but these methods lack specificity due to cross-reactivity and matrix interferences. We developed and validated an isotopic dilution-liquid chromatography-tandem mass spectrometry (ID-LC-MS/MS) method for the simultaneous measurement of cortisol, corticosterone, 11deoxycortisol, androstenedione, deoxycorticosterone (DOC), testosterone, 17OHprogesterone, dehydroepiandrosterone (DHEA) and progesterone in serum, and compared it to routine immunoassays employed in our laboratory. We also established adult reference intervals in 416 healthy subjects. Methods: $0.9 \mathrm{ml}$ of serum were spiked with labelled internal standards (IS) and extracted on C18 cartridges. Eluate was injected into a two-dimensional LC-system, purified in a perfusion column and separated on a C8 column during a 21 min gradient run. Analytes were revealed by atmospheric pressure chemical ionization (APCI) followed by multiple reaction monitoring (MRM) analysis.

Results: Of the four immunoassays compared with the ID-LC-MS/MS method, only the results of ElecsysE170 for cortisol, testosterone in males and progesterone $>1 \mathrm{ng} / \mathrm{ml}$ were in agreement with ID-LC-MS/MS. ElecsysE170 for testosterone in females and progesterone $<1 \mathrm{ng} / \mathrm{ml}$, Immulite 2000 for androstenedione, DSL-9000 for DHEA and 170HP Bridge for 170Hprogesterone, respectively, showed poor agreement. Reference intervals and steroid age and fertility related fluctuations were established. Conclusion: Our ID-LC-MS/MS method proved to be reliable and sensitive in revealing steroid circulating concentrations in adults and in highlighting the limits of routine immunoassays at low concentrations.
\end{abstract}

(c) 2010 Elsevier Inc. All rights reserved.

\footnotetext{
Abbreviations: ID-LC-MS/MS, isotopic dilution-liquid chromatography-tandem mass spectrometry; DOC, deoxycorticosterone; DHEA, dehydroepiandrosterone; IS, internal standard; APCI, atmospheric pressure chemical ionization; MRM, multiple reaction monitoring; GC-MS, gas chromatography-mass spectrometry; DHEA-S, DHEA-sulphate; BSA, bovine serum albumin; HPLC, high pressure liquid chromatography; SPE, solid phase extraction; QC, quality control; BMI, body mass index; CAD, collision activated dissociation; CUR, curtain gas; LLOQ, lower limit of quantification; $\mathrm{S} / \mathrm{N}$, signal to noise ratio; LOD, limit of detection; IR, ion ratio; IQR, interquartile range; $\mathrm{CI}$, confidence interval; $\mathrm{MW}$, molecular weight; RT, retention time; DP, declustering potential; CE, collision energy; CXP, cell exit potential; $S_{y / x}$, standard deviation of residuals; $\mathrm{M}$, males; $\mathrm{F}$, females; pre-M, pre-menopausal females; postM, post-menopausal females; s.d., standard deviation.

* Corresponding author at: Endocrinology Unit, S.Orsola-Malpighi Hospital, Via Massarenti 9, 40138 Bologna, Italy. Tel.: +39 051 6363009; fax: +39 0516363080.

E-mail address: uberto.pagotto@unibo.it (U. Pagotto).
}

\section{Introduction}

Steroid measurement remains a challenge for the endocrinological community. Since the RIA breakthrough 40 years ago [1,2], immunoassays have remained the most common tool to characterize the pathophysiological states of circulating steroids. RIA combined with extraction and chromatographic purification steps have been attributed acceptable specificity and good sensitivity. Nowadays, most methodologies are dominated by automated chemiluminescent or electro-chemiluminescent immunoassay platforms and by semi-automated RIAs. These assays offer simplicity and high throughput, key-factors for a large routine application, but due to cross-reactivity and matrix interferences they lack specificity [3]. Poor validation and standardization data are often provided for these methods, causing a huge variability among dif- 
ferent kits and among different laboratories employing the same kits and leading to significant variations in reference intervals from one commercial assay to another $[4,5]$. These drawbacks limit diagnostic accuracy, appropriate treatment and follow-up in clinical praxis, and preclude epidemiological multicenter studies [6-9].

Gas chromatography-mass spectrometry (GC-MS) was first introduced in the 1960s and improved in the 1980s to represent the gold standard method for steroid analysis [10-12]. However, this technique requires complex time-consuming derivatization procedures for sample pre-treatment, limiting its application in routine clinical practice. Conversely, ID-LC-MS/MS is an innovative technology combining the high selectivity and sensitivity of mass spectrometry with the versatility of liquid chromatography. ID-LC-MS/MS allows reliable, simultaneous quantification of a wide panel of steroids in a broad concentration range with high throughput capabilities [13]. These advantages have yielded insights into biochemical changes and more useful clinical data, but a re-definition of age and sex specific reference intervals is urgently needed. Many ID-LC-MS/MS methods for steroid measurement have been proposed in recent years [14-22], often performing multi-hormone analysis in a short chromatographic run and requiring less-demanding sample preparation. However, a good pre-analytical treatment and a careful chromatographic separation of frequently occurring isobaric analytes are still needed for sensitive and specific steroid monitoring in complex matrices like serum or urine [23].

This study describes the development and validation of an IDLC-MS/MS method for the simultaneous measurement of nine serum steroids of clinical relevance, comparing it with six routinely used immunoassays. In addition, we evaluated the steroid profile of 416 healthy normal weight drug-free subjects, aged 18-89 years, analyzing the influence of age and fertility status on steroid levels.

\section{Materials and methods}

\subsection{Chemicals}

The following compounds were used: cortisol, corticosterone, 11 deoxycortisol, androstenedione, DOC, testosterone, 17OHprogesterone, DHEA, progesterone, 21deoxycortisol, epitestosterone, DHEA-sulphate (DHEA-S) and cortisone (Steraloids, Newport, RI); d4-cortisol, d8-corticosterone, d2-11deoxycortisol, d5-testosterone, d8-170Hprogesterone, d2-DHEA and d9-progesterone (CDN Isotopes, Pointe Claire, Canada); ${ }^{13} \mathrm{C} 2$-testosterone (Cambridge Isotope Laboratories, Andover, MA); bovine serum albumin (BSA), prednisone and prednisolone (Sigma-Aldrich, St. Louis, MO); betamethasone disodium phosphate (Defiante Farmaceutica, Madeira, Portugal); dexamethasone 21phosphate disodium salt (Visufarma, Rome, Italy); methylprednisolone acetate (Pfizer, New York City, NY); triamcinolone acetonide (Bristol-Myers Squibb, New York City, NY). Gradient grade methanol and zinc sulphate hepta-hydrated were from Merck (Darmstadt, Germany); ultrapure water was produced by MilliQ Gradient A10 system (Millipore, Volketswil, Switzerland). Steroid-free serum was from MP Biomedicals (Solon, $\mathrm{OH}$ ). The solid phase extraction (SPE) cartridges were IST Isolute C18 $100 \mathrm{mg}, 1 \mathrm{~cm}^{3}$ from Biotage (Uppsala, Sweden). Reference material was from the Reference Institute for Bioanalytics (Bonn, Germany).

\subsection{Standard solutions, calibrators and in-house quality control (QC) samples}

Stock solutions were prepared in methanol for each standard and isotopically labelled IS at different concentrations in the $\mathrm{mg} / \mathrm{ml}$ range. Working solutions were at $100 \mathrm{ug} / \mathrm{ml}$ for cortisol, progesterone and ISs, and at $10 \mathrm{ug} / \mathrm{ml}$ for other analytes. A stock calibrator was prepared by mixing each standard to obtain the following concentrations: cortisol, $500 \mathrm{ng} / \mathrm{ml}$; DHEA and progesterone, $50 \mathrm{ng} / \mathrm{ml}$; corticosterone, androstenedione and testosterone, $20 \mathrm{ng} / \mathrm{ml} ; 11$ deoxycortisol, DOC and $170 H$ progesterone, $10 \mathrm{ng} / \mathrm{ml}$. An eight-point calibration curve was prepared by serial dilution of the stock calibrator in 4\% BSA. The BSA solution represented the "zero" calibration point. The working IS solution was a mixture of d4-cortisol $50 \mathrm{ng} / \mathrm{ml}$, d8corticosterone and d2-11deoxycortisol $5 \mathrm{ng} / \mathrm{ml}$, d2-DHEA $3 \mathrm{ng} / \mathrm{ml}$, ${ }^{13} \mathrm{C} 2$-testosterone $2 \mathrm{ng} / \mathrm{ml}$ and $\mathrm{d} 8-170 H$ progesterone $1 \mathrm{ng} / \mathrm{ml}$, d9progesterone $10 \mathrm{ng} / \mathrm{ml}$. Stock solutions, working solutions and calibrators were stored at $-20^{\circ} \mathrm{C}$. Three in-house QCs were prepared by generating a serum pool used as the low level and by adding standard solutions to obtain the medium and high levels for each analyte. Calibrators for androstenedione determination at 0.5 and $5 \mathrm{ng} / \mathrm{ml}$ of Immulite2000 (Siemens Healthcare Diagnostics, Deerfield, IL), at $0.98,2.5,9.8$ and $25.0 \mathrm{ng} / \mathrm{ml}$ of DSL-9000 RIA kit (Webster, TX) and at $0.1,0.35,2.0$ and $10.0 \mathrm{ng} / \mathrm{ml}$ of $170 H P$ Bridge RIA kit (Adaltis, Guidonia, Italy) were measured by ID-LC-MS/MS as such for calibration assessment.

\subsection{Specimens}

De-identified samples for method comparison were collected among sera from S.Orsola-Malpighi Hospital routine laboratory. Healthy volunteers, males and females aged 19-89 years, were recruited for reference interval estimation, after having given their informed consent, at the local health service of the town of Massa Lombarda. The study was approved by the local Ethical Committee. Body mass index (BMI) ranged between 18.1 and $25.0 \mathrm{~kg} / \mathrm{m}^{2}$. The inclusion criteria were: body weight stability in the last 3 months, complete sexual development and menstrual cycle regularity in fertile women. Subjects taking drugs (except for antipyretic or antiinflammatory compounds), or presenting endocrine, hepatic, renal, tumoral, autoimmune, cardiovascular, hematologic, neurologic or psychiatric diseases, sleep disorders, or allergies requiring treatment were excluded. Between 8 and 10 a.m., subjects were infused with saline for $10 \mathrm{~min}$ before blood collection in a Vacuette $Z$ serum beads clot activator (Greiner Bio-One, Kremsmunster, Austria); samples were centrifuged at $2000 \times g$ for $10 \mathrm{~min}$ at room temperature and sera were stored in $1.5 \mathrm{ml}$ polypropylene tubes at $-20^{\circ} \mathrm{C}$ until analysis.

\subsection{Sample preparation}

Serum samples and in-house QCs were thawed and vortexed. Liophilic reference samples were reconstituted with $3 \mathrm{ml} \mathrm{H}_{2} \mathrm{O}$ and gently mixed at room temperature for $60 \mathrm{~min}$. For each sample, curve calibrator, immunoassay calibrators, and QCs 900 ul were transferred to $12 \mathrm{~mm} \times 75 \mathrm{~mm}$ glass tubes before addition of $1 \mathrm{ml}$ of $\mathrm{MeOH}:\left[\left(\mathrm{ZnSO}_{4}\right)\left(\mathrm{H}_{2} \mathrm{O}\right)_{7}\right.$ aqueous solution $\left.8.9 \%, \mathrm{w} / \mathrm{v}\right]=80: 20$ containing IS. After $3 \mathrm{~min}$ vortex, tubes were centrifuged for $10 \mathrm{~min}$ at $2000 \times g$ at room temperature. Supernatants were transferred on the SPE cartridge previously activated with $1 \mathrm{ml}$ of $\mathrm{MeOH}$ and conditioned with $1 \mathrm{ml}$ of $\mathrm{H}_{2} \mathrm{O}$. After washing with $3 \mathrm{ml}$ of $\mathrm{H}_{2} \mathrm{O}$ cartridges were eluted with $1 \mathrm{ml}$ of $\mathrm{MeOH}$. Eluates were dried under nitrogen stream, reconstituted with $0.3 \mathrm{ml}$ of $50 \% \mathrm{MeOH}$ and transferred into glass vials and placed into a Series 200 Autosampler thermostated at $10^{\circ} \mathrm{C}$ (PerkinElmer, Waltham, MA).

\subsection{On-line purification and LC separation}

Two hundred microliters were injected into a two-dimensional LC-system consisting in a modular HPLC Series 200 by PerkinElmer, further purified on perfusion column POROS 

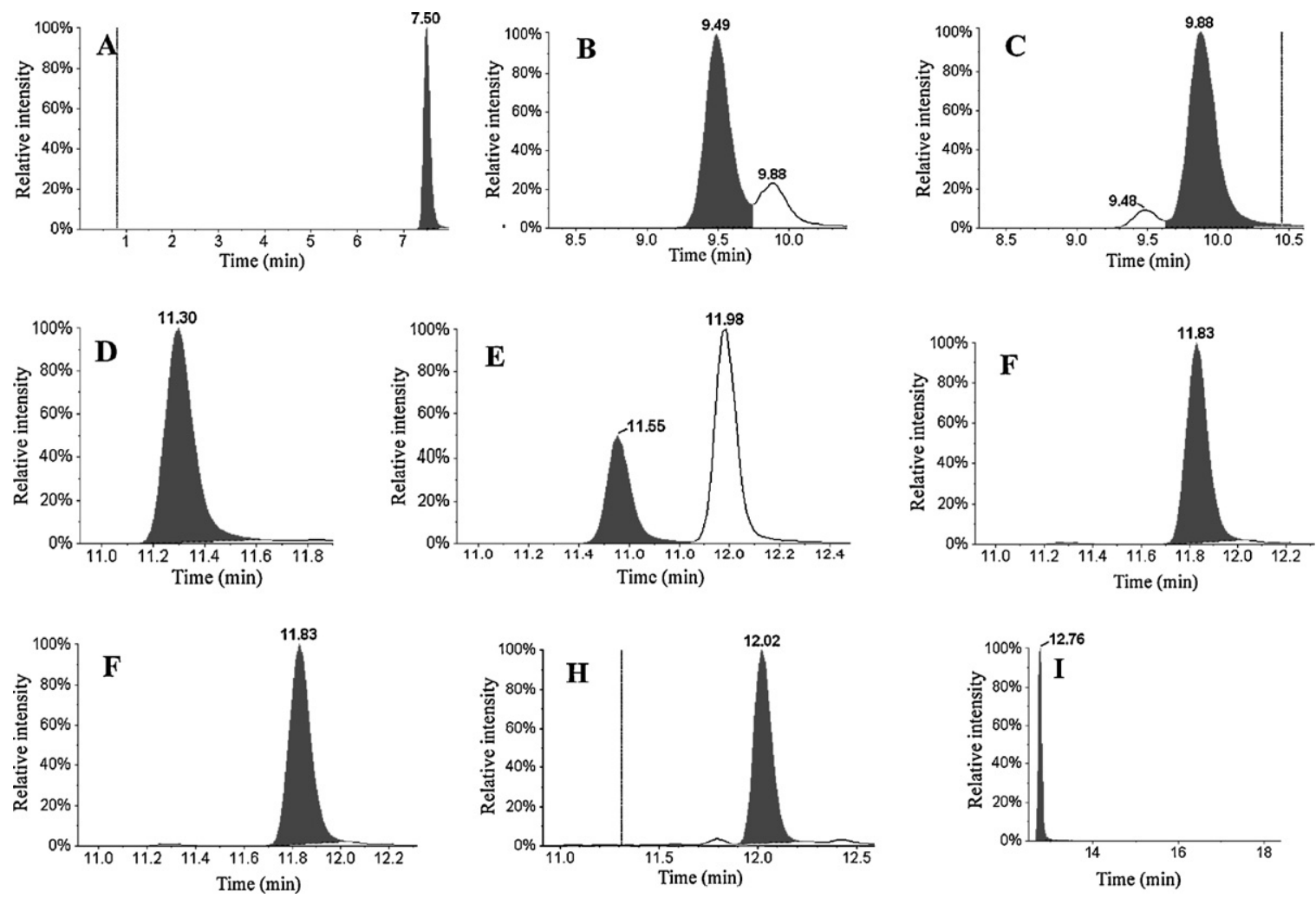

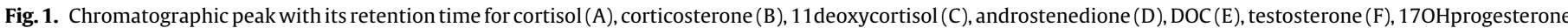
$(\mathrm{G}), \operatorname{DHEA}(\mathrm{H})$ and progesterone (I).

$\mathrm{R} 1 / 202.1 \mathrm{~mm} \times 30 \mathrm{~mm}$ by Applied Biosystems (Foster City, CA). After washing with $10 \% \mathrm{MeOH}$ at $3 \mathrm{ml} / \mathrm{min}$ for $1 \mathrm{~min}$, the sample was back-flushed to the analytical column Luna RP-C8 $100 \mathrm{~mm} \times 4.6 \mathrm{~mm}, 5 \mathrm{um}$ (Phenomenex, Torrance, CA) equipped with an RP-C8 $4 \mathrm{~mm} \times 2 \mathrm{~mm}, 5 \mathrm{um}$ guard column, through a ten-port switching valve (VICl, Houston, TX) at $0.750 \mathrm{ml} / \mathrm{min}$ of an eluent made with $98 \%$ solvent $\mathrm{A}(20 \% \mathrm{MeOH})$ and $2 \%$ solvent $\mathrm{B}$ (100\% $\mathrm{MeOH})$. The 21 min gradient run program started with $45 \%$ solvent B and at min 8.5 a linear gradient to $100 \%$ in 2 min was activated with a subsequent $2 \mathrm{~min}$ washing step and a $6.5 \mathrm{~min}$ reequilibration step to the initial conditions. Chromatographic peaks are shown in Fig. 1. Chromatographic resolution was achieved for the isobaric steroid pairs (corticosterone and 11deoxycortisol, DOC and 17OHprogesterone, testosterone and DHEA) and for the steroids, like testosterone and androstenedione, which differ in their molecular weight by only 2 amu and which could interfere through the specific +2 amu-isotopomer of one analyte over the mono-isotopic form of the other.

\subsection{Mass spectrometry detection}

Mass spectrometric measurements were performed by an API 4000-QTrap (AB-Sciex, Toronto, Canada) working in triplequadrupole mode. Quantification was performed by the MRM mode, choosing for each analyte two specific transitions, one for the quantitative assessment (the "quantifier") and one for confirmation (the "qualifier"). The parameters pertaining to the MRM transitions were optimized by infusing standard solutions at concentrations ranging from $100 \mathrm{ng} / \mathrm{ml}$ to $10 \mathrm{ug} / \mathrm{ml}$, into the Turbo-V source through an infusion pump set at $10 \mathrm{ul} / \mathrm{min}$ in addition to a makeup flow of $50 \% \mathrm{MeOH}$ at $400 \mathrm{ul} / \mathrm{min}$ (Table 1 ). The APCI probe operated with a Corona discharge current of $3 \mathrm{uA}$ in positive ion mode. Collision activated dissociation (CAD) gas was nitrogen set at a pressure of 10 mTorr and the other parameter settings were: probe temperature $400^{\circ} \mathrm{C}$, curtain gas (CUR) 30 psi, nebulising gas $30 \mathrm{psi}$. To maximize the dwell time for each targeted analyte, LC-run monitoring was divided into four periods: 0.0-8.3 min; 8.4-10.9 min; 11.0-12.5 min; $12.6-21.0 \mathrm{~min}$. Unit mass resolution was set at both Q1 and Q3.

\subsection{Quantitation}

Data processing and quantitation were performed by Analyst 1.4.2 software package by AB-Sciex. Calibration was done through linear regression: concentrations for each analyte were back calculated by interpolation on the respective regression curve.

\subsection{ID-LC-MS/MS method validation}

Four per cent BSA was chosen as a suitable matrix for standard calibration points. The isotopic dilution quantitation method was assessed as follows: d4-cortisol was used as IS for cortisol, d8corticosterone for corticosterone; d2-11deoxycortisol for 11deoxycortisol; ${ }^{13} \mathrm{C} 2$-testosterone for androstenedione, testosterone and DHEA; d8-17OHprogesterone for DOC, 170Hprogesterone and progesterone. D5-testosterone, d2-DHEA and d9-progesterone were discarded since their unsuitability for the remarkable signal instability with the APCI source (likely more than on the electrospray source), and for the cross-interference on the unlabelled standard steroid transitions; analogous phenomena were also reported by Vogeser and co-workers in a recent publication [24]. A $1 / x$ weighting regression was chosen to ensure higher accuracy and precision at the low concentration end of the curve. Linearity was achieved for three-four orders of magnitude. The lower limit of quantification (LLOQ) was determined on five replicates as the lowest concentration exhibiting a signal to noise ratio $(\mathrm{S} / \mathrm{N})$ above ten, with an accuracy between 80 and $120 \%$ of the true value and with CV below $20 \%$. The limit of detection (LOD) was determined as the lowest 
Table 1

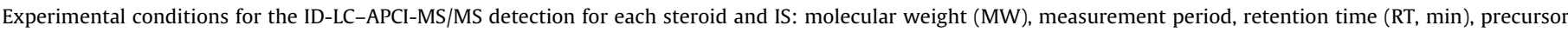

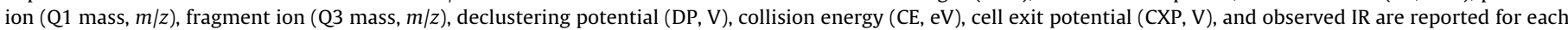
targeted compound.

\begin{tabular}{|c|c|c|c|c|c|c|c|c|c|c|}
\hline Analyte & MW & Period & RT & Transition & Q1 mass & Q3 mass & $\mathrm{DP}$ & $\mathrm{CE}$ & CXP & IR \\
\hline \multirow[t]{2}{*}{ Cortisol } & 362.46 & 1 & 7.4 & Quantifier & 363.2 & 121.2 & 60 & 45 & 3 & 4.3 \\
\hline & & & & Qualifier & 363.2 & 267.4 & 60 & 35 & 5 & \\
\hline D4-cortisol & 366.46 & 1 & 7.4 & IS & 367.3 & 97.1 & 50 & 45 & 3 & \\
\hline \multirow[t]{2}{*}{ Corticosterone } & 346.46 & 2 & 9.5 & Quantifier & 347.1 & 121.0 & 76 & 45 & 9 & 2.1 \\
\hline & & & & Qualifier & 347.1 & 97.1 & 76 & 45 & 5 & \\
\hline D8-corticosterone & 354.46 & 2 & 9.4 & IS & 355.4 & 125.4 & 88 & 45 & 5 & \\
\hline \multirow[t]{2}{*}{ 11Deoxycortisol } & 346.46 & 2 & 9.9 & Quantifier & 347.2 & 109.1 & 82 & 45 & 5 & 1.1 \\
\hline & & & & Qualifier & 347.2 & 97.0 & 82 & 45 & 5 & \\
\hline D2-11deoxycortisol & 348.46 & 2 & 9.9 & IS & 349.4 & 97.1 & 75 & 45 & 3 & \\
\hline \multirow[t]{2}{*}{ Androstenedione } & 286.41 & 3 & 11.3 & Quantifier & 287.4 & 97.0 & 78 & 30 & 3 & 1.4 \\
\hline & & & & Qualifier & 287.4 & 109.0 & 78 & 40 & 5 & \\
\hline \multirow[t]{2}{*}{ DOC } & 330.5 & 3 & 11.6 & Quantifier & 331.4 & 109.1 & 80 & 40 & 4 & 1.1 \\
\hline & & & & Qualifier & 331.4 & 97.0 & 80 & 30 & 4 & \\
\hline \multirow[t]{2}{*}{ Testosterone } & 288.42 & 3 & 11.8 & Quantifier & 289.2 & 97.1 & 78 & 35 & 3 & 1.0 \\
\hline & & & & Qualifier & 289.2 & 109.1 & 78 & 35 & 5 & \\
\hline${ }^{13} \mathrm{C} 2$-testosterone & 290.41 & 3 & 11.8 & IS & 291.4 & 111.1 & 74 & 35 & 5 & \\
\hline \multirow[t]{2}{*}{ 170HProgesterone } & 330.46 & 3 & 12.0 & Quantifier & 331.1 & 97.0 & 70 & 40 & 3 & 0.9 \\
\hline & & & & Qualifier & 331.1 & 109.3 & 70 & 45 & 5 & \\
\hline D8-170HProgesterone & 338.46 & 3 & 11.9 & IS & 339.5 & 100.1 & 50 & 45 & 7 & \\
\hline \multirow[t]{2}{*}{ DHEA } & 288.42 & 3 & 12.1 & Quantifier & 271.3 & 197.2 & 55 & 25 & 3 & 3.0 \\
\hline & & & & Qualifier & 271.3 & 213.3 & 55 & 25 & 4 & \\
\hline \multirow[t]{2}{*}{ Progesterone } & 314.46 & 4 & 12.8 & Quantifier & 315.6 & 97.1 & 80 & 30 & 3 & 1.1 \\
\hline & & & & Qualifier & 315.6 & 109.1 & 80 & 40 & 4 & \\
\hline
\end{tabular}

concentration exhibiting a S/N above three. Data are summarized in Table 2.

Interference with other endogenous and exogenous steroids was investigated. Amounts of 21deoxycortisol, epitestosterone, DHEA-S, cortisone, prednisone and prednisolone were injected into the LC-MS/MS system: traces shown in Supplemental Fig. 1 highlight the good immunity of the monitored steroids from the massive presence of the others. Triamcinolone acetonide, methylprednisolone, dexamethasone and betamethasone, were spiked at $500 \mathrm{ng} / \mathrm{ml}$ into the QC samples and processed as unknown samples: no interference over calculated concentrations of monitored steroids was found. No interference by serum matrix non-steroid compounds was shown by injecting steroid-free serum samples. Intensity ratios between quantifier and qualifier transitions (ion ratio, IR) were monitored in each sample to check for any unexpected interference. Sample IR was accepted within $20 \%$ of the calibrator IR.

Method imprecision was assessed on six replicates per day of in-house QC samples at low, medium and high concentrations (intra-assay), and on six different days (inter-assay). CV was accepted below $15 \%$ in the intra-assay and below $20 \%$ in inter-assay.
Trueness, expressed as the percentage of found concentration over nominal concentration, was assessed in the low, medium and high range by reference certified sera for cortisol, testosterone, $170 H$ progesterone and progesterone, and by in-house validation for corticosterone, 11deoxycortisol, androstenedione, DOC and DHEA, by spiking gravimetrically determined quantities of pure standards in steroid-free serum, previously checked for absence of steroids above the LOD. Data are summarized in Table 3.

Sensitivity was also evaluated in serum matrix, as reported for LLOQ, by spiking minimal amounts of analyte standards in steroidfree serum in three replicates (Table 2).

Ion suppression was investigated by spiking equal amounts of standard analytes either on pre-extracted BSA solutions or steroid-free serum, to exclude any procedural losses from the yield calculation. Analyte peak areas were compared in BSA and in steroid-free serum, and both were compared to peak areas of pure standards (Supplemental Table 1). Negligible deviation from $100 \%$, denoting absence of suppression, was observed. A post-column infusion of a mixture containing the nine steroids at concentrations suitable for generating measurable steady-state signals was performed during injections of blank and steroid-free serum

Table 2

ID-LC-MS/MS method calibration curve and sensitivity $\left(S_{y / x}\right.$ : standard deviation of residuals).

\begin{tabular}{|c|c|c|c|c|c|c|c|c|c|c|c|}
\hline & \multirow{2}{*}{$\begin{array}{l}\text { Linear range } \\
(\mathrm{ng} / \mathrm{ml})\end{array}$} & \multirow[t]{2}{*}{ Slope } & \multirow[t]{2}{*}{ Intercept } & \multirow[t]{2}{*}{$S_{y / x}$} & \multirow[t]{2}{*}{$r^{2}$} & \multicolumn{4}{|l|}{ LLOQ } & \multirow{2}{*}{$\begin{array}{l}\text { LOD (pg on } \\
\text { column) }\end{array}$} & \multirow{2}{*}{$\begin{array}{l}\text { Sensitivity in } \\
\text { serum matrix } \\
(\mathrm{ng} / \mathrm{ml})\end{array}$} \\
\hline & & & & & & $\mathrm{ng} / \mathrm{ml}$ & $\mathrm{S} / \mathrm{N}$ & CV \% & Accuracy \% & & \\
\hline Cortisol & $0.244-500.0$ & $0.0551 \pm 0.0022$ & $0.0050 \pm 0.0022$ & 1.673 & 0.9997 & 0.2440 & 49 & 9.0 & 99.3 & 4.8 & 0.244 \\
\hline Corticosterone & $0.039-20.0$ & $0.2652 \pm 0.0213$ & $-0.0003 \pm 0.0019$ & 0.100 & 0.9995 & 0.0391 & 13 & 3.4 & 95.9 & 4.0 & 0.313 \\
\hline 11Deoxycortisol & $0.019-10.0$ & $0.1748 \pm 0.0106$ & $0.0025 \pm 0.0014$ & 0.075 & 0.9993 & 0.0195 & 14 & 15.3 & 94.1 & 2.0 & 0.078 \\
\hline Androstenedione & $0.019-20.0$ & $0.4577 \pm 0.0139$ & $0.0032 \pm 0.0013$ & 0.047 & 0.9998 & 0.0195 & 14 & 13.2 & 100.7 & 2.3 & 0.039 \\
\hline DOC & $0.019-10.0$ & $0.8857 \pm 0.0451$ & $-0.0006 \pm 0.0018$ & 0.032 & 0.9994 & 0.0195 & 11 & 9.6 & 107.8 & 2.5 & 0.078 \\
\hline Testosterone & 0.019-20.0 & $0.4922 \pm 0.0145$ & $0.0158 \pm 0.0027$ & 0.110 & 0.9993 & 0.0195 & 11 & 6.2 & 94.2 & 2.8 & 0.019 \\
\hline 17OHProgesterone & $0.010-10.0$ & $0.9012 \pm 0.0649$ & $0.0011 \pm 0.0023$ & 0.033 & 0.9996 & 0.0098 & 11 & 8.9 & 107.5 & 1.7 & 0.078 \\
\hline DHEA & $0.195-50.0$ & $0.0164 \pm 0.0036$ & $0.0002 \pm 0.0001$ & 0.348 & 0.9995 & 0.1953 & 10 & 11.2 & 97.4 & 29.2 & 0.781 \\
\hline Progesterone & $0.024-50.0$ & $0.4527 \pm 0.0273$ & $0.0035 \pm 0.0024$ & 0.117 & 0.9999 & 0.0244 & 29 & 9.3 & 103.4 & 1.2 & 0.049 \\
\hline
\end{tabular}




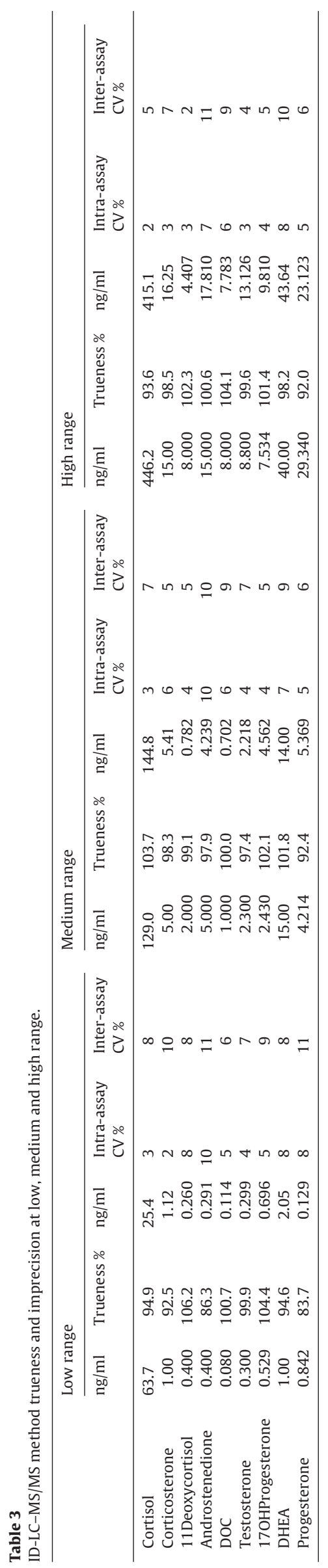

extracts. Negligible increase or decrease in MRM scan intensities were observed, denoting a negligible ion suppression effect.

\subsection{Immunoassays}

Cortisol, testosterone and progesterone were measured by electro-chemiluminescence immunoassay on the Modular Analytics ElecsysE170 by Roche Diagnostics (Mannheim, Germany); androstenedione by the solid-phase, competitive chemiluminescent enzyme immunoassay Immulite2000; DHEA by DSL-9000 RIA and 170 Hprogesterone by $170 \mathrm{HP}$ Bridge RIA.

\subsection{Data analysis and statistics}

\subsubsection{Method comparison}

ID-LC-MS/MS method was compared with the immunoassay measurements for cortisol $(n=159)$, testosterone $(n=162)$ progesterone $(n=85)$, androstenedione $(n=137)$, DHEA $(n=143)$ and $170 H$ progesterone $(n=99)$. To avoid bias attributable to different sensitivity limits, results below the sensitivity limit were excluded. Calibration agreement between Immulite2000, DSL-9000 and 17OHP Bridge and ID-LC-MS/MS was assessed by measuring kit calibrators by ID-LC-MS/MS. No comparison was performed for corticosterone, 11 deoxycortisol and DOC since no routine methods were available in the reference laboratory.

Non-normally distributed variables were compared by the Mann-Whitney test and all data are expressed as median and interquartile range (IQR). The Deming regression was applied to account for the imprecision of both methods [25]. Bland and Altman plots were drawn for agreement estimation, representing the percentage difference between the methods against the mean [26].

\subsubsection{Estimation of reference intervals}

Median values and non-parametric 2.5th and 97.5th centiles [27] were estimated in males $(n=217)$ and females $(n=199)$. The female group was subdivided according to fertility status into premenopausal ( $n=134$ of whom 51 women in the follicular phase [days $1-10])$, and post-menopausal $(n=65)$ subgroups. Reference intervals in luteal phase were not evaluated because of the small number of samples available. The effect of age on steroid level was evaluated by the Spearman regression analysis.

Data analysis was performed on MedCalc v9.3.7.0 (Mariakerke, Belgium).

\section{Results}

\subsection{Method comparison study}

By the Mann-Whitney comparison, ElecsysE170 and IDLC-MS/MS methods provided non-different results for testosterone in males and for progesterone above $1 \mathrm{ng} / \mathrm{ml}$. Lower median values were obtained by ID-LC-MS/MS compared with ElecsysE170 for determination of cortisol $(-16 \%, p=0.0052)$, testosterone in females $(-26 \%, p=0.0080)$ and progesterone below $1 \mathrm{ng} / \mathrm{ml}$ ( $-84 \%, p<0.0001)$. ID-LC-MS/MS, compared to Immulite2000, DSL-9000 and 170HP Bridge for measurement of androstenedione, DHEA and 170Hprogesterone, respectively, provided significantly lower results $(p<0.0001)$, immunoassay medians being 2-3-fold higher than ID-LC-MS/MS medians (Supplemental Table 2).

Deming regression graphs, slope and intercept coefficients with respective standard errors and correlation coefficient between immunoassays and ID-LC-MS/MS are reported in Fig. 2. In males, the slope and the intercept obtained from the regression between testosterone results by ElecsysE170 and ID-LC-MS/MS were not different from one and zero, parameters of the curve of best 

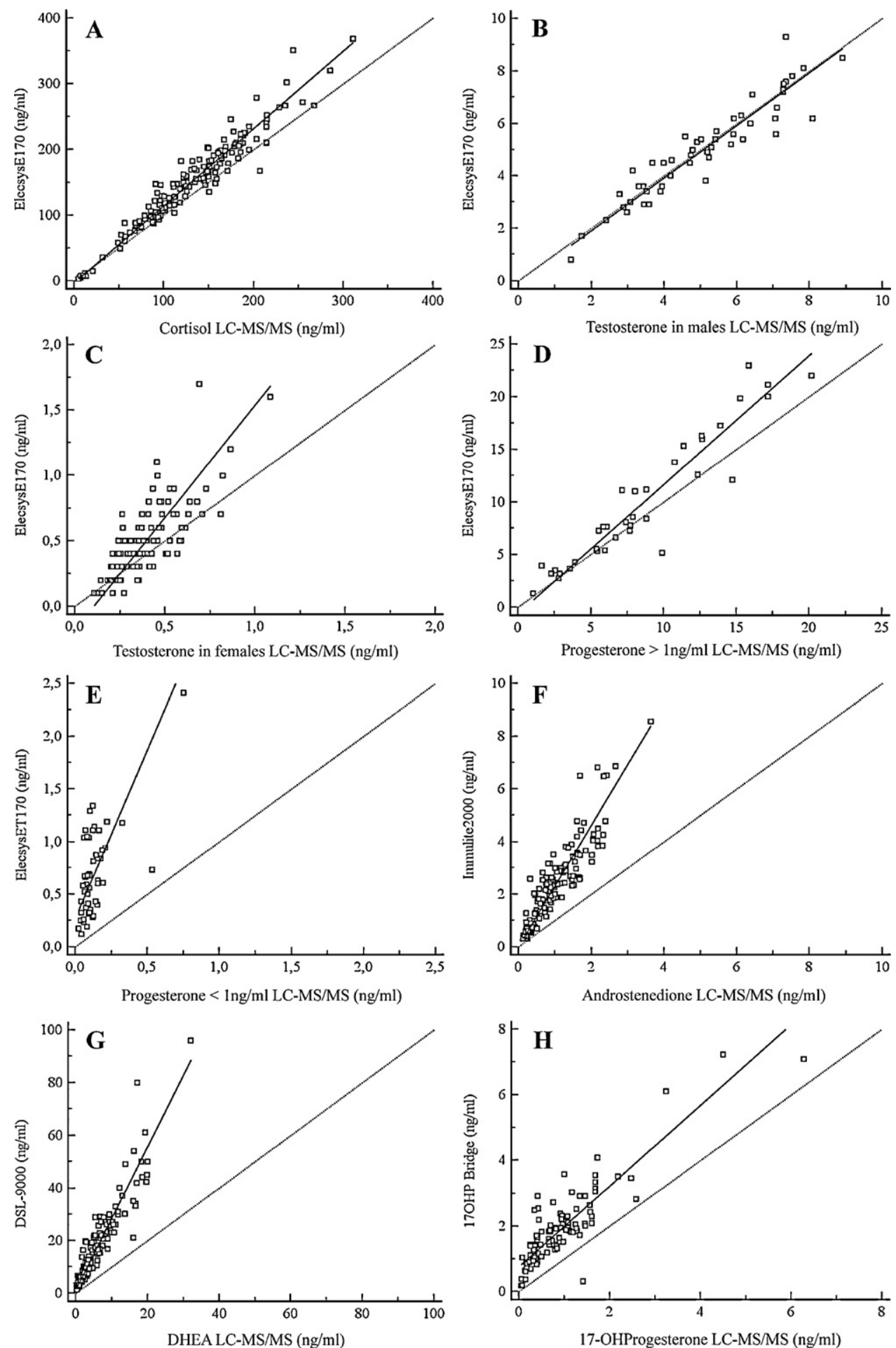

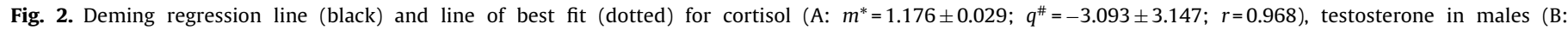

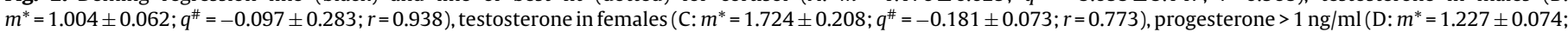

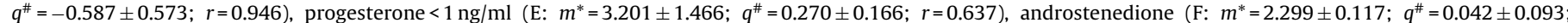

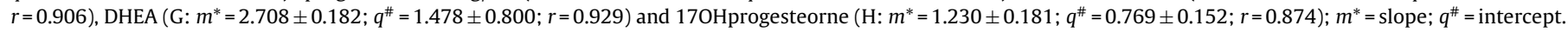

fit, respectively. A slight but statistically elevated slope and an intercept not different from zero were obtained for cortisol and progesterone above $1 \mathrm{ng} / \mathrm{ml}$ [95\% confidence interval (CI): 1.119-1.233 and 1.077-1.378, respectively].

Testosterone in females and progesterone below $1 \mathrm{ng} / \mathrm{ml}$ exhibited elevated slope coefficients (95\%Cl: $1.311-2.137$ and $0.253-6.149$, respectively) and testosterone in females had a nega- tive intercept coefficient ( $95 \% \mathrm{CI}$ : -0.326 to -0.036$)$. The regression between ID-LC-MS/MS and Immulite2000, DSL-9000 and 17OHP Bridge, for the determination of androstenedione, DHEA and $170 H$ progesterone provided significantly elevated slopes $(95 \% \mathrm{CI}$ : $2.068-2.530 ; 2.348-3.068 ; 0.870-1.589$, respectively), but only $170 H$ progesterone regression provided a non-negligible positive intercept coefficient (95\%CI: 0.468-1.071). 

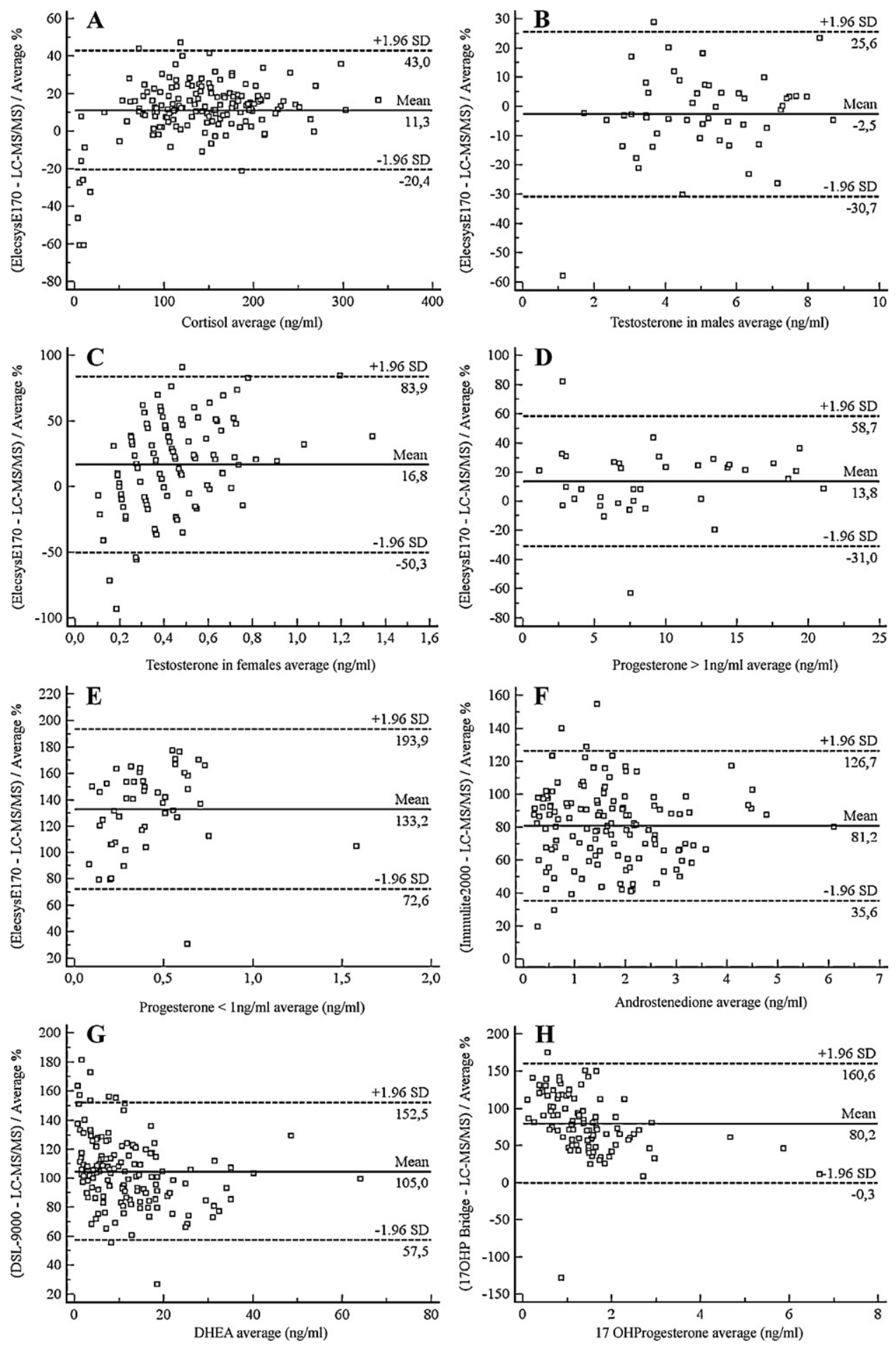

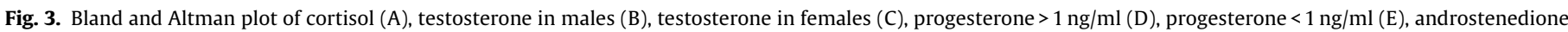

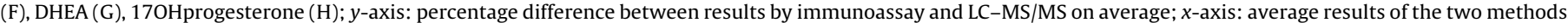

By the Bland and Altman agreement estimation we found that among the four immunoassays compared to ID-LC-MS/MS method, only ElecsysE170 method for all analytes, except progesterone below $1 \mathrm{ng} / \mathrm{ml}$, provided an acceptable mean difference. However, only for testosterone in males the mean difference was not different from $0 \%(95 \% \mathrm{Cl}$ : $-6.6 \%$ to $1.5 \%)$. For all other analytes worse agreement was obtained, with mean differences ranging from $80.2 \%$ of $170 H$ progesterone to $133.2 \%$ of progesterone below $1 \mathrm{ng} / \mathrm{ml}$. Furthermore, despite the notable width, agreement intervals for androstenedione, DHEA, 17OHprogesterone and progesterone below $1 \mathrm{ng} / \mathrm{ml}$ were entirely located on the positive side of the graph, with only one case in which measurement of 170 Hprogesterone was higher for immunoassay compared to ID-LC-MS/MS (Fig. 3). These data indicated a good accuracy and calibration of ElecsysE170 for the determination of cortisol, testosterone and progesterone, analytes for which trueness of the ID-LC-MS/MS method was confirmed against GC-MS certified sera. Nevertheless, Deming regression, correlation of coefficients and Bland and Altman analysis showed that ElecsysE170 is affected by cross-reactivity at low ranges of testosterone and progesterone. 
Table 4

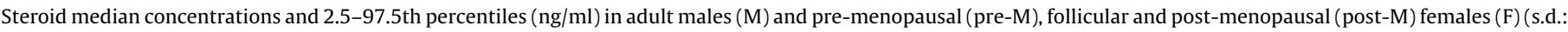
standard deviation). Blood samples were taken from 8:00 to 10:00 a.m.

\begin{tabular}{|c|c|c|c|c|}
\hline & M & F pre-M & F follicular & F post-M \\
\hline Age & $18-89$ & $18-54$ & $18-54$ & $45-86$ \\
\hline BMI (mean \pm s.d.) & $23.2 \pm 1.7$ & $21.7 \pm 2.0$ & $21.8 \pm 2.0$ & $23.2 \pm 1.7$ \\
\hline $\mathrm{N}$ & 217 & 134 & 51 & 65 \\
\hline Hormone & Median (2.5-97.5P) & Median (2.5-9 7.5P) & Median (2.5-97.5P) & Median (2.5-97.5P) \\
\hline Cortisol & $119.9(45.7-199.4)$ & $101.5(47.4-199.7)$ & $113.1(40.5-199.8)$ & $114.7(56.9-180.4)$ \\
\hline Corticosterone & $3.23(0.46-12.60)$ & $2.62(0.62-11.85)$ & $2.89(0.45-11.94)$ & $2.74(0.68-8.54)$ \\
\hline 11Deoxycortisol & $0.325(0.086-1.094)$ & $0.239(<1.081)$ & $0.249(<1.345)$ & $0.284(0.082-0.838)$ \\
\hline Androstenedione & $0.571(0.262-1.263)$ & $0.748(0.277-1.638)$ & $0.727(0.308-1.602)$ & $0.299(0.095-0.773)$ \\
\hline Testosterone & $5.34(2.82-8.18)$ & $0.248(0.104-0.454)$ & $0.248(0.116-0.431)$ & $0.147(0.077-0.392)$ \\
\hline DHEA & $4.97(1.40-14.28)$ & $5.09(1.19-18.93)$ & $5.68(2.03-27.04)$ & $2.45(0.80-6.60)$ \\
\hline 170HProgesterone & $1.095(0.415-2.542)$ & $0.578(0.152-2.266)$ & $0.411(0.161-0.947)$ & $0.209(<0.527)$ \\
\hline Progesterone & $0.078(<0.189)$ & $0.203(<17.816)$ & $0.093(<1.673)$ & $<0.049(<0.080)$ \\
\hline
\end{tabular}

The results given by the measurement of 170HP Bridge calibrators by ID-LC-MS/MS assessed the good calibration of this kit, trueness of the ID-LC-MS/MS method being confirmed against GC-MS certified sera: accuracy at points $0.1,0.35,2.0$ and $10.0 \mathrm{ng} / \mathrm{ml}$ was $92.8 \%, 101.3 \%, 96.7 \%$ and $101.6 \%$, respectively. The data above, together with the poor correlation coefficient (Fig. 2), the discrepancies observed in the Mann-Whitney comparison (Supplemental Table 2) and the results obtained by the Bland and Altman analysis (Figs. 2 and 3), clearly explain that the overestimation by $170 H P$ Bridge RIA is due to the cross-reactivity and not to the miscalibration. By measuring DHEA in DSL-9000 calibrators by IDLC-MS/MS, we found an accuracy of 53.9\%, 48.5\%, $46.2 \%$ and $55.5 \%$ at points $0.98,2.5,9.8$ and $25 \mathrm{ng} / \mathrm{ml}$, respectively. Such a miscalibration can only in part explain the huge overestimation shown by the Mann-Whitney comparison and by the regression analysis. The wide agreement range (57.5-152.5\%) obtained in the Bland and Altman plots suggested the presence of a non-proportional bias, probably due to the cross-reactivity of the antibody employed in the assay. The accuracy obtained by the measurement of androstenedione in Immulite 2000 calibrators was $133.1 \%$ and $82.5 \%$ at 0.5 and at $5.0 \mathrm{ng} / \mathrm{ml}$, respectively. Similarly to DSL-9000, this miscalibration may explain only part of the overestimation exhibited by Immulite2000 (Fig. 1, Supplemental Table 2), but both the 2.5 -fold increase observed in the Mann-Whitney comparison and the wide range of agreement observed in the Bland and Altman analysis (35.6-126.7\%) suggest a further contribute due to cross-reactivity (Fig. 3).

\subsection{Reference intervals}

Reference intervals and median values are listed in Table 4. The reference interval for DOC was not set because, despite a LLOQ of $19.5 \mathrm{pg} / \mathrm{ml}$, the sensitivity in serum matrix $(78 \mathrm{pg} / \mathrm{ml})$ proved unsatisfactory for the measurement of this hormone. A single measurement of corticosterone, testosterone and 170Hprogesterone, two of DHEA, four of 11 deoxycortisol and 28 of progesterone were discarded because of the questionable IRs.

In females, a single measurement of corticosterone, androstenedione and DHEA, 13 of 11 deoxycortisol ( $8 \%$ of pre-menopausal and $3 \%$ of post-menopausal), three of $170 H$ progesterone and 65 of progesterone, 46 of them in the post-menopausal subgroup (71\%), whereas in males four measurements of 11deoxycortisol, and 39 of progesterone were below the sensitivity limit.

In males, androstenedione, testosterone, DHEA, 170Hprogesterone and progesterone exhibited a significant negative correlation with age (Supplemental Fig. 2) [rho $=-0.236(p=0.0005)$; $-0.224(p=0.0010) ;-0.576(p<0.0001) ;-0.185(p=0.0066)$ and $-0.268(p=0.0002)$, respectively]. In the pre-menopausal subgroup an age-dependent decrease of androstenedione, testosterone and DHEA was also observed (Supplemental Fig. 3) [rho $=-0.334$ $(p=0.0001) ;-0.232(p=0.0076)$ and $-0.466(p<0.0001)$, respectively] and it continued for DHEA, but not for androstenedione and testosterone, in the post-menopausal subgroup ( $\mathrm{rho}=-0.282$, $p=0.024)$, whereas an age-dependent increase was observed for cortisol and 11deoxycortisol: rho $=0.369(p=0.0031)$ and 0.401 $(p=0.0015)$, respectively. In the post-menopausal subgroup, levels of androstenedione, testosterone and DHEA were significantly lower than pre-menopausal levels, and levels of $170 H$ progesterone and progesterone lower than follicular phase levels $(p<0.0001$ for all). No significant changes were observed for cortisol and 11 deoxycortisol.

\section{Discussion}

We developed a sensitive ID-LC-MS/MS method for the simultaneous measurement of nine serum steroids. This is a powerful tool in clinical praxis to depict various pathophysiological alterations in steroid secretion in a single run. By using a sample volume higher than those reported in other published multi-analyte ID-LC-MS/MS methods $(15-20 ; 22)$, we were able to provide a general better sensitivity for the 9 hormones, determined not only as LLOQ but also in a complex matrix, more similar to the real samples, as steroid free serum.

Such high sensitivity is very much needed for the careful definition of the lower reference limits, although DOC detection in the healthy population is still unsatisfying. However, in the routine application of our method, less sample volume may be used if the determination of steroids whose circulating levels are close to method sensitivity, like 11deoxycortisol, corticosterone, DOC, DHEA and progesterone, are not required. By increasing sample volume, noise and interferences may also be of relevance, and a second purification step on the perfusion column after SPE concentration was therefore adopted. The double purification guaranteed ruggedness across the analysis of many samples per run in several runs per week, helping to keep the system stable and clean and minimizing matrix interference. The chromatographic conditions guaranteed an adequate resolving power for isobaric compounds like corticosterone and 11deoxycortisol, DOC and $170 H$ progesterone, respectively, and for the isotopic pattern cross-interferences between androstenedione and testosterone. The method also exhibited a good immunity from matrix components, as proved by European certified sera analyses and by the in-house validation.

The comparison of our ID-LC-MS/MS method with routine immunoassays employed in the clinical laboratory revealed a good agreement for ElecsysE170 in determining elevated levels of cortisol, testosterone in males and progesterone above $1 \mathrm{ng} / \mathrm{ml}$. However, the ElecsysE170 performance was not reliable for low 
levels of testosterone and progesterone both for lack of specificity and general overestimation, proving severely inadequate in depicting females' health status, especially for testosterone, thereby confirming other reports $[5,8,28]$. The huge overestimation obtained by Immulite 2000 and DSL- 9000 in measuring androstenedione and DHEA, respectively, is not only due to miscalibration between ID-LC-MS/MS and these immunoassays, but also to a sub-optimal specificity that may complicate evaluations in clinical borderline situations. In agreement with previous reports [16,29-31], we also demonstrated that the overestimation exhibited by $170 \mathrm{HP}$ Bridge should be attributed to cross-reactivity, and finally to a severe lack of specificity in $170 H$ progesterone measurement, being the ID-LC-MS/MS accuracy verified against reference material. Our present study took into account only one immunoassay per analyte; however we cannot exclude that other immunometric platforms or RIAs may perform better and give more consistent results.

Few steroid ID-LC-MS/MS reference intervals on adult populations have been published to date, and the topic remains a matter of debate. We therefore provided preliminary reference intervals for a wide pattern of steroids in an adult male and female population by analyzing 416 healthy drug-free normal-weight subjects. Our data showed a strong age-dependent decrease of androgens both in males and, to a greater extent, in pre-menopausal females. DHEA showed the highest rate of decrease in both sexes including postmenopausal females, whereas testosterone and androstenedione remained stable in post-menopausal women. The post-menopausal group showed an age-dependent increase in cortisol and 11deoxycortisol. In the male group, 170 Hprogesterone and progesterone declined with age, but the sensitivity limit of progesterone probably masks an even higher extent of this tendency. In females, reference intervals were examined in the follicular phase, but more subjects distributed through the menstrual cycle are needed for the complete reference intervals of these hormones during physiological fluctuations.

Some discrepancies between our values and literature data could be due to sample collection: our subjects were saline infused for $10 \mathrm{~min}$ before blood withdrawal to avoid stress-related alterations in the glucocorticoid cascade. In particular, our cortisol and corticosterone intervals are lower than those reported by others, whereas 11 deoxycortisol and 170 Hprogesterone are generally higher $[14,15,19,21]$. Our data on $170 H$ progesterone intervals in females cannot be compared with a previous work as no information on fertility was provided [15]. The intervals we obtained for androstenedione are in agreement with those reported by Kushnir in 2010 [22]. Male testosterone values are similar to those previously published [22,32-34], whereas the upper limit found in pre-menopausal females is generally lower than those reported [22,32-34]. Since circulating androgen levels are strongly age-related, comparison with cohorts of different ages is difficult. Multicenter studies involving a larger number of subjects grouped for decades are needed to establish age-related intervals and steroid trends. Not only the sampling procedures, but also ethnicity or statistical calculation of reference limits may account for differences among reports. A further confounding factor is the inclusion of subjects with BMI above 25. We took particular care to exclude overweight or obese subjects to define as normal values that are modified by subtle changes in body weight. However, the main cause of poor agreement among literature reports lies in the miscalibration and generally poor standardization of IDLC-MS/MS methods, since reference procedures and matrix-based calibrators for most steroids are not available, and in-house validation alone is not sufficient to achieve a consensus [12,35]. To date, few studies have compared data from different ID-LC-MS/MS laboratories, and most focused on testosterone measurement [36,37]. Further comparative studies of steroid measurement are urgently needed to gain advances in biomedical research and enhance clinical care.

\section{Acknowledgements}

This research was supported by grants from European Union (REPROBESITY FPVII-223713 and NEUROFAST FPVII-KBBE-2009-3245009). We thank Fondazione Cassa di Risparmio, Bologna, Italy for supporting the Centre of Applied Biomedical Research and the Progetto Regione-Università of Region Emilia Romagna for the support to this study. We also thank Dr. P. Chieco for the laboratory organization, Dr. A.M. Morselli-Labate for the help in statistical analysis, Dr. M. Baldassarre and Dr. M. Mezzullo for the artwork and Dr. A. Collins for language editing of the manuscript.

\section{Appendix A. Supplementary data}

Supplementary data associated with this article can be found, in the online version, at doi:10.1016/j.steroids.2010.11.005.

\section{References}

[1] Abraham GE. Solid-phase radioimmunoassay of estradiol-17 beta. J Clin Endocrinol Metab 1969;29:866-70.

[2] Furuyama S, Mayes DM, Nugent CA. A radioimmunoassay for plasma testosterone. Steroids 1970;16:415-28.

[3] Bock JL. The new era of automated immunoassay. Am J Clin Pathol 2000;113:628-46.

[4] Matsumoto AM, Bremner WJ. Serum testosterone assays-accuracy matters [Editorial]. J Clin Endocrinol Metab 2004;89:520-4.

[5] Wang C, Catlin DH, Demers LM, Starcevic B, Swerdloff RS. Measurement of total serum testosterone in adult men: comparison of current laboratory methods versus liquid chromatography-tandem mass spectrometry. J Clin Endocrinol Metab 2004;89:534-43.

[6] Potischman N, Falk RT, Laiming VA, Siiteri PK, Hoover RN. Reproducibility of laboratory assays for steroid hormones and sex hormone-binding globulin. Cancer Res 1994;54:5363-7.

[7] Herold DA, Fitzgerald RL. Immunoassays for testosterone in women: better than a guess? Clin Chem 2003;49:1250-1.

[8] Taieb J, Mathian B, Millot F, Patricot MC, Mathieu E, Queyrel N, et al. Testosterone measured by 10 immunoassays and by isotope-dilution gas chromatography-mass spectrometry in sera from 116 men, women and children. Clin Chem 2003;49:1381-95.

[9] Stanczyk FZ, Lee JS, Santen RJ. Standardization of steroid hormone assays: why, how, and when? Cancer Epidemiol Biomarker 2007;16:1713-9.

[10] Wolthers BG, Kraan GP. Clinical applications of gas chromatography and gas chromatography-mass spectrometry of steroids. J Chromatogr A 1999;843:247-74.

[11] Krone N, Hughes BA, Lavery GG, Stewart PM, Arlt W, Shackleton CH. Gas chromatography/mass spectrometry (GC/MS) remains a pre-eminent discovery tool in clinical steroid investigations even in the era of fast liquid chromatography tandem mass spectrometry (LC/MS/MS). J Steroid Biochem Mol Biol 2010:22 [Epub ahead of print], Apr 22.

[12] Stanczyk FZ, Clarke NJ. Advantages and challenges of mass spectrometry assays for steroid hormones. J Steroid Biochem Mol Biol 2010;12 [Epub ahead of print], May.

[13] Vogeser M, Parhofer KG. Liquid chromatography tandem-mass spectrometry (LC-MS/MS)-technique and applications in endocrinology. Exp Clin Endocrinol Diabetes 2007; 115:559-70.

[14] Kushnir MM, Neilson R, Roberts WL, Rockwood AL. Cortisol and cortisone analysis in serum and plasma by atmospheric pressure photoionization tandem mass spectrometry. Clin Biochem 2004;37:357-62.

[15] Kushnir MM, Rockwood AL, Roberts WL, Pattison EG, Owen WE, Bunker AM et al. Development and performance evaluation of a tandem mass spectrometry assay for 4 adrenal steroids. Clin Chem 2006;52:1559-67.

[16] Rauh M, Gröschl M, Rascher W, Dörr HG. Automated, fast and sensitive quantification of 17 alpha-hydroxy-progesterone, androstenedione and testosterone by tandem mass spectrometry with on-line extraction. Steroids 2006;71:450-8.

[17] Guo T, Taylor RL, Singh RJ, Soldin SJ. Simultaneous determination of 12 steroids by isotope dilution liquid chromatography-photospray ionization tandem mass spectrometry. Clin Chim Acta 2006;372:76-82.

[18] Janzen N, Sander S, Terhardt M, Peter M, Sander J. Fast and direct quantification of adrenal steroids by tandem mass spectrometry in serum and dried blood spots. J Chromatogr B Analyt Technol Biomed Life Sci 2008;861:117-22.

[19] Carvalho VM, Nakamura OH, Vieira JG. Simultaneous quantitation of seven endogenous C-21 adrenal steroids by liquid chromatography tandem mass spectrometry in human serum. J Chromatogr B Analyt Technol Biomed Life Sci 2008;872:154-61. 
[20] Ceglarek U, Kortz L, Leichtle A, Fiedler GM, Kratzsch J, Thiery J. Rapid quantification of steroid patterns in human serum by on-line solid phase extraction combined with liquid chromatography-triple quadrupole linear ion trap mass spectrometry. Clin Chim Acta 2009;401:114-8.

[21] Rossi C, Calton L, Hammond G, Brown HA, Wallace AM, Sacchetta P, et al Serum steroid profiling for congenital adrenal hyperplasia using liquid chromatography-tandem mass spectrometry. Clin Chim Acta 2010;411:222-8.

[22] Kushnir MM, Blamires T, Rockwood AL, Roberts WL, Yue B, Erdogan E, et al. Liquid chromatography-tandem mass spectrometry assay for androstenedione, dehydroepiandrosterone, and testosterone with pediatric and adult reference intervals. Clin Chem 2010;56:1138-47.

[23] Vogeser M, Seger C. A decade of HPLC-MS/MS in the routine clinical laboratory-goals for further developments. Clin Biochem 2008;41:649-62.

[24] Vogeser M, Seger C. Pitfalls associated with the use of liquid chromatographytandem mass spectrometry in the clinical laboratory. Clin Chem 2010;56:1234-44.

[25] Linnet K. Performance of Deming regression analysis in case of misspecified analytical error ratio in method comparison studies. Clin Chem 1998;44:1024-31.

[26] Bland JM, Altman DG. Measuring agreement in method comparison studies. Stat Methods Med Res 1999;8:135-60.

[27] Morselli Labate AM, Rusticali AG. La determinazione dei valori di riferimento in laboratorio. 2. Valutazione matematica dell'intervallo di riferimento. Ligand Q 1986;5:77-80.

[28] Moal V, Mathieu E, Reynier P, Malthièry Y, Gallois Y. Low serum testosterone assayed by liquid chromatography-tandem mass spectrometry. Comparison with five immunoassay techniques. Clin Chim Acta 2007;386:12-9.
[29] Turpeinen U, Itkonen O, Ahola L, Stenman UH. Determination of 17alphahydroxyprogesterone in serum by liquid chromatography-tandem mass spectrometry and immunoassay. Scand J Clin Lab Inv 2005;65:3-12.

[30] Etter ML, Eichhorst J, Lehotay DC. Clinical determination of 17hydroxyprogesterone in serum by LC-MS/MS: comparison to Coat-A-Count RIA method. J Chromatogr B Analyt Technol Biomed Life Sci 2006;840:69-74.

[31] Brossaud J, Barat P, Gualde D, Corcuff JB. Cross reactions elicited by serum 17-OH progesterone and 11-deoxycortisol in cortisol assays. Clin Chim Acta 2009;407:72-4.

[32] Turpeinen U, Linko S, Itkonen O, Hamalainen E. Determination of testosterone in serum by liquid chromatography-tandem mass spectrometry. Scand J Clin Lab Inv 2008;68:50-7.

[33] Shiraishi S, Lee PW, Leung A, Goh VH, Swerdloff RS, Wang C. Simultaneous measurement of serum testosterone and dihydrotestosterone by liquid chromatography-tandem mass spectrometry. Clin Chem 2008;54:1855-63.

[34] Salameh WA, Redor-Goldman MM, Clarke NJ, Reitz RE, Caulfield MP. Validation of a total testosterone assay using high-turbulence liquid chromatography tandem mass spectrometry: total and free testosterone reference ranges. Steroids 2010;75:169-75.

[35] Demers LM. Testosterone and estradiol assays: current and future trends. Steroids 2008;73:1333-8.

[36] Thienpont LM, Van Uytfanghe K, Blincko S, Ramsay CS, Xie H, Doss RC, et al. State-of-the-art of serum testosterone measurement by isotope dilution-liquid chromatography-tandem mass spectrometry. Clin Chem 2008;54:1290-7.

[37] Vesper HW, Bhasin S, Wang C, Tai SS, Dodge LA, Singh RJ, et al. Interlaboratory comparison study of serum total testosterone [corrected] measurements performed by mass spectrometry methods. Steroids 2009;74:498-503. 